\title{
Genome-wide identification of the SPL gene family in Tartary Buckwheat (Fagopyrum tataricum) and expression analysis during fruit development stages
}

Moyang Liu ${ }^{1,2+}$, Wenjun Sun ${ }^{1 \dagger}$, Zhaotang Ma', Li Huang ${ }^{1}$, Qi Wu' ${ }^{1}$, Zizhong Tang ${ }^{1}$, Tongliang Bu ${ }^{1}$, Chenglei Li ${ }^{1}$ and Hui Chen ${ }^{1 *}$ D

\begin{abstract}
Background: SPL (SQUAMOSA promoter binding protein-like) is a class of plant-specific transcription factors that play important roles in many growth and developmental processes, including shoot and inflorescence branching, embryonic development, signal transduction, leaf initiation, phase transition, and flower and fruit development. The SPL gene family has been identified and characterized in many species but has not been well studied in tartary buckwheat, which is an important edible and medicinal crop.

Results: In this study, 24 Fagopyrum tataricum SPL (FtSPL) genes were identified and renamed according to the chromosomal distribution of the FtSPL genes. According to the amino acid sequence of the SBP domain and gene structure, the SPL genes were divided into eight groups (group I to group VII) by phylogenetic tree analysis. A total of 10 motifs were detected in the tartary buckwheat SPL genes. The expression patterns of 23 SPL genes in different tissues and fruits at different developmental stages (green fruit stage, discoloration stage and initial maturity stage) were determined by quantitative real-time polymerase chain reaction (qRT-PCR).

Conclusions: The tartary buckwheat genome contained 24 SPL genes, and most of the genes were expressed in different tissues. QRT-PCR showed that FtSPLs played important roles in the growth and development of tartary buckwheat, and genes that might regulate flower and fruit development were preliminarily identified. This work provides a comprehensive understanding of the SBP-box gene family in tartary buckwheat and lays a significant foundation for further studies on the functional characteristics of FtSPL genes and improvement of tartary buckwheat crops.
\end{abstract}

Keywords: Tartary buckwheat, FtSPL, Genome, Fruit development, Expression patterns

\section{Background}

Tartary buckwheat originated in Southwest China and is currently grown in Southwest China and the Himalayan region, as well as in Japan, Canada and other countries. As a widely cultivated medicinal and edible crop, tartary buckwheat is rich in complete proteins with a wellbalanced composition of essential amino acids and is rich in beneficial soluble fiber and phytochemicals [1-4].

\footnotetext{
* Correspondence: chenhui@sicau.edu.cn

${ }^{+}$Moyang Liu and Wenjun Sun contributed equally to this work.

${ }^{1}$ College of Life Science, Sichuan Agricultural University, Ya'an, China

Full list of author information is available at the end of the article
}

Tartary buckwheat fruits are rich in rutin, which has been reported to prevent liver damage [5]. The development and ripening of tartary buckwheat fruit are important stages of its growth and development. Transcription factors (TFs) play an indispensable role in regulating the growth and development of plants. At present, the auxin response factor gene family has been identified in tartary buckwheat, and the potential role of Fagopyrum tataricum ARF2 (FtARF2) on the fruit size of tartary buckwheat has been studied $[6,7]$. Because the SQUAMOSA promoter binding protein $(\mathrm{SBP})$-like $(S P L)$ gene regulates inflorescence branching and grain development, it is important to

(C) The Author(s). 2019 Open Access This article is distributed under the terms of the Creative Commons Attribution 4.0 International License (http://creativecommons.org/licenses/by/4.0/), which permits unrestricted use, distribution, and 
explore the potential functions of FtSPL genes to understand the regulation of the growth and development of tartary buckwheat.

The SBP-box is an important transcription factor family that regulates growth and development in plants. It was first isolated from the cDNA library of Antirrhinum majus inflorescences and was named for its ability to recognize and bind to the promoter of SQUAMOSA (SQ-UA) $[8,9]$. SPL is a general term for a class of transcription factors similar to SBP-box. The SPL genes encode a highly conserved SBP domain containing approximately 76 amino acid residues, including two tandem zinc fingers (Cys-Cys-His-Cys and Cys-Cys-Cys-His), and possess a nuclear localization signal (NLS) at the C-terminus $[10,11]$. Studies have shown that SPL TFs exist widely in plants and play an important role in many aspects of plant growth and development, including regulating flower formation and late flower development, controlling plant transformation from vegetative growth to reproductive growth, regulating leaf morphogenesis, and responding to environmental signals [12-15].

With the release of plant genome data, the genome-wide identification and analysis of the whole SPL gene family have been carried out in many plants, such as Arabidopsis thaliana (A. thaliana) [16, 17], Solanum lycopersicum (S. lycopersicum) [18], Glycine max (G. max) [19], Vitis vinifera (V. vinifera) [20], Malus domestica Borkh (M. domestica) [21], Ricinus communis L (R. communis L) [22], Zea mays L (Z. mays) [23], Capsicum annuum L (C. annuum) [24], and Salvia miltiorrhiza (S. miltiorrhiza) [25]. In A. thaliana, the 16 A. thaliana SPL (AtSPL) genes were divided into 8 groups according to the amino acid sequence of the SBP domain: AtSPL7 (I group), AtSPL1/12/14/16 (II group), AtSPL8 (III group), AtSPL6 (IV group), AtSPL2/10/ 11 (V group), AtSPL3/4/5 (VI group), AtSPL13 (VII group), and AtSPL9/15 (VII group). The functions of these SPL genes in A. thaliana have also been identified, and they play an important role in leaf, flower and shoot development $[15,26,27]$. At present, many SPL genes have been isolated and identified in other plants, but studies on the SPL proteins in tartary buckwheat have not been conducted, and the functions of these proteins are not clear.

In this study, we comprehensively analyzed the gene structures, motif compositions, chromosomal locations, and gene duplications of 24 SPL genes on the basis of the recently completed genome sequence of tartary buckwheat and compared the evolutionary relationship of tartary buckwheat with $A$. thaliana, Beta vulgaris (B. vulgaris), G. max, $S$. lycopersicum, $V$. vinifera, and Oryza sativa (O. sativa). Compared with the identification of SPL family in other plants, this study not only analyzed the grouping, gene structure, chromosome localization, tandem and fragment replication of FtSPL gene family, but also analyzed the evolutionary relationship of FtSPL genes in multiple species, including multiple species phylogenetic tree, motif composition and collinearity analysis among them. In addition, we studied the spatial expression of SPL genes in different tissues and their expression patterns during tartary buckwheat fruit development. Through global expression analysis, the roles of specific SPL gene family members in different biological processes of tartary buckwheat were determined. This study comprehensively analyzed SPL gene families in tartary buckwheat, not only providing valuable information for screening important $S P L$ genes in tartary buckwheat growth and development, but also providing methods for mining SPL families in other plants.

\section{Results}

Identification of the FtSPL genes in tartary buckwheat

In this study, we extracted the SPL genes from the tartary buckwheat genome by the two BLASTp methods, and we identified 24 SPL genes from tartary buckwheat after removing the redundant sequences. We renamed the $S P L$ genes based on their location on the chromosomes of tartary buckwheat (Additional file 2 Table S1). From the location information of the SPL genes, we saw that all SPL genes were located in the nucleus (Additional file 2 Table S1). The predicted SPL proteins of tartary buckwheat varied greatly in length and molecular weight (MV). The tartary buckwheat SPL genes encoded proteins ranging from 100 (FtPinG0006337300.01) to 1004 (FtPinG0000377600.01) amino acids (aa) in length and from 11.7 (FtPinG00063373 00.01) to 110.77 (FtPinG0000377600.01) kDa in MV. The isoelectric points (PIs) of the proteins ranged from 5.69 (FtPinG0000377600.01) to 9.75 (FtPinG0001287900.01) (Additional file 2 Table S1).

\section{Multiple sequence alignment, phylogenetic analysis, and classification of the FtSPL genes}

Multiple sequence alignment with the SPL full-length protein sequence showed that the SBP domain was basically conserved in specific positions, such as the CQQC sequence (235-238 amino acid), SCR sequence (253-256 amino acid) and RRR sequence (264-267 amino acid). Almost all sequences contained two zinc finger-like structures, Zn-1, Zn-2 and a highly conserved nuclear localization signal (NLS) (Additional file 1 Figure S1).

To investigate the phylogenetic relationships of the $S P L$ genes in tartary buckwheat, 24 tartary buckwheat $S P L$ genes and $17 \mathrm{~A}$. thaliana SPL genes were used to construct a neighbor-joining (N-J) phylogenetic tree using MEGA 6.0 software. From the phylogenetic tree, we saw that the FtSPL genes were divided into eight subgroups (I to VII). There was only one member from tartary buckwheat in group I and group VII, five members from tartary buckwheat in group II and group VI, three members from tartary buckwheat in group IV and group V, two members from tartary buckwheat in group III, and Group VII had four members from tartary buckwheat (Fig. 1). 


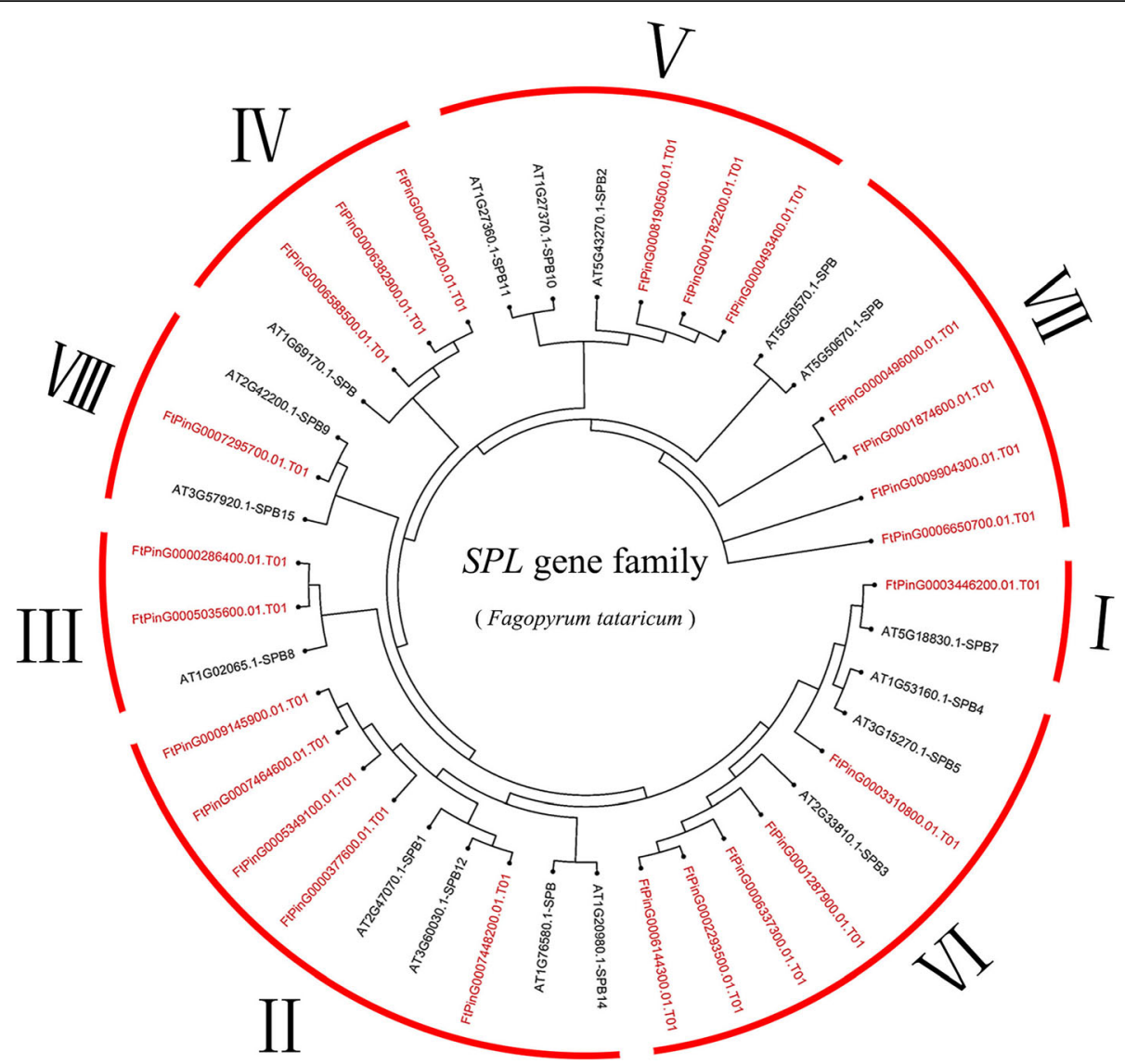

Fig. 1 Unrooted phylogenetic tree representing the relationships among 24 SPL genes of tartary buckwheat and Arabidopsis. The genes in tartary buckwheat are marked in red, while those in Arabidopsis are marked in black.

\section{Gene structure and motif composition of the FtSPL gene family}

When a phylogenetic tree based on the full-length predicted FtSPL protein sequences was constructed, the structural diversity of the tartary buckwheat SPL genes was examined, and these proteins were also roughly divided into eight subgroups (Fig. 2). The diversity of the genetic structure may be a mechanism to promote the evolution of multigene families. We compared the number and location of the exons and introns in the SPL gene sequences of tartary buckwheat and further explored the structural diversity of the FtSPL genes. From Fig. 2b, we saw that the genes in group II contained the largest number of exons, of which FtPinG0007448200.01 contained 11 exons, while other genes contained 10 exons. The FtSPL genes in group VI all had two exons, which was the lowest number of exons among these subgroups. In general, the number of exons within the same subgroup was similar, although the locations of the exons were different.

Further analysis showed that there was a conserved SBP domain in the N-terminus of all FtSPL proteins and a conserved ANK domain only in the C-terminus of members of group II (FtPinG0007448200.01/377600.01/5349100.01/
7464600.01/9145900.01) (Fig. 2b). We used MEME software to detect the motif compositions of the entire sequences, including the conserved domains in all FtSPL proteins, to explore the diversity of the motifs in each protein (Fig. 2c). A total of 10 different motifs were detected in the tartary buckwheat FtSPL genes and named motif 1 to motif 10. The length and sequences of the 10 motifs are listed in Table S3. The number of conserved motifs in each FtSPL protein ranged from 1 to 10. All FtSPL proteins except FtPinG0006850700.01 and FtPinG0001874600.01 contained motif 1 and motif 2, while those in group II contained all the motifs. Twelve FtSPL proteins only had motif 1 and motif 2, and FtPinG0001874600.01 only contained motif 2 . In short, members belonging to the same subgroup had similar a gene structure and motif composition and tended to gather together in the phylogenetic trees.

\section{Chromosomal distribution, gene duplication and synteny analysis of the FtSPL genes}

The chromosome mapping of the FtSPL genes was performed using the latest tartary buckwheat genome database. A total of 24 SPL genes were unevenly distributed in eight tartary buckwheat linkage groups (LGs) (Fig. 3). 


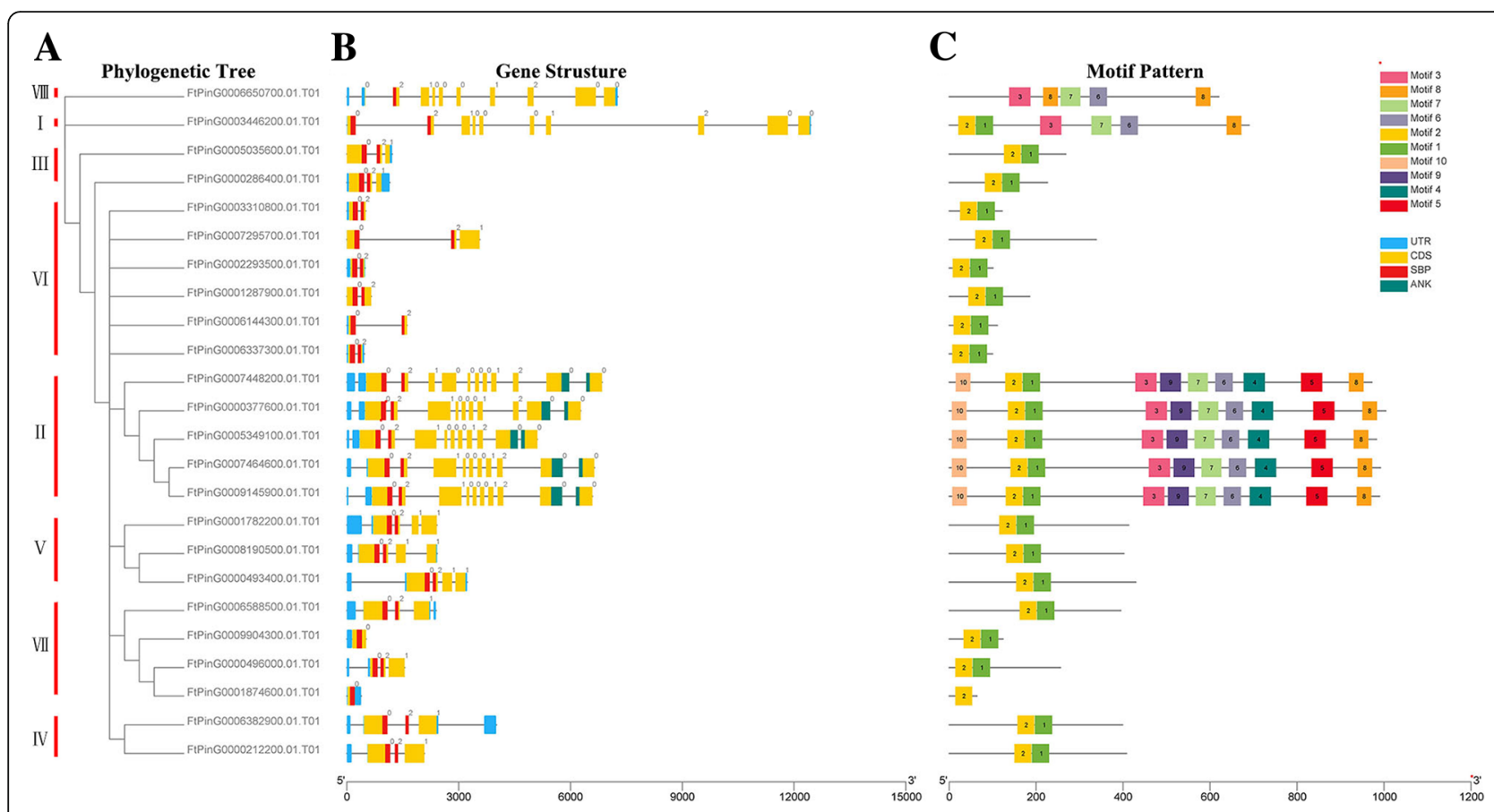

Fig. 2 Phylogenetic relationships, gene structures and architectures of the conserved protein motifs of the SPL genes from tartary buckwheat. a The phylogenetic tree was constructed based on the full-length sequences of tartary buckwheat SPL proteins using Geneious R11 software. $\mathbf{b}$ Exon-intron structures of tartary buckwheat SPL genes. Yellow boxes indicate untranslated 5'- and 3'-regions; blue boxes indicate exons; and black lines indicate introns. The SBP domain is highlighted by a red box. The number indicates the phases of the corresponding introns. $\mathbf{c}$ The motif compositions of the tartary buckwheat SPL proteins. The motifs, numbered 1-10, are displayed in different colored boxes. The sequence information for each motif is provided in Table S2. The protein length can be estimated using the scale at the bottom.

There was only one gene (FtPinG0009145900.01) in LG 7. There were four genes in LG1, LG2 and LG8, which was the largest number of genes.

Gene duplication events play an indispensable role in the production of new functions and in gene expansion. Therefore, we analyzed the duplication events of the SPL genes in the tartary buckwheat genome. There were no tandem duplication events in the tartary buckwheat SPL genes, but there were 6 pairs of segmental duplicates (Fig. 4). There are many homologous genes on the different chromosomes of tartary buckwheat, which support the high level of conservation of the SPL gene family. In

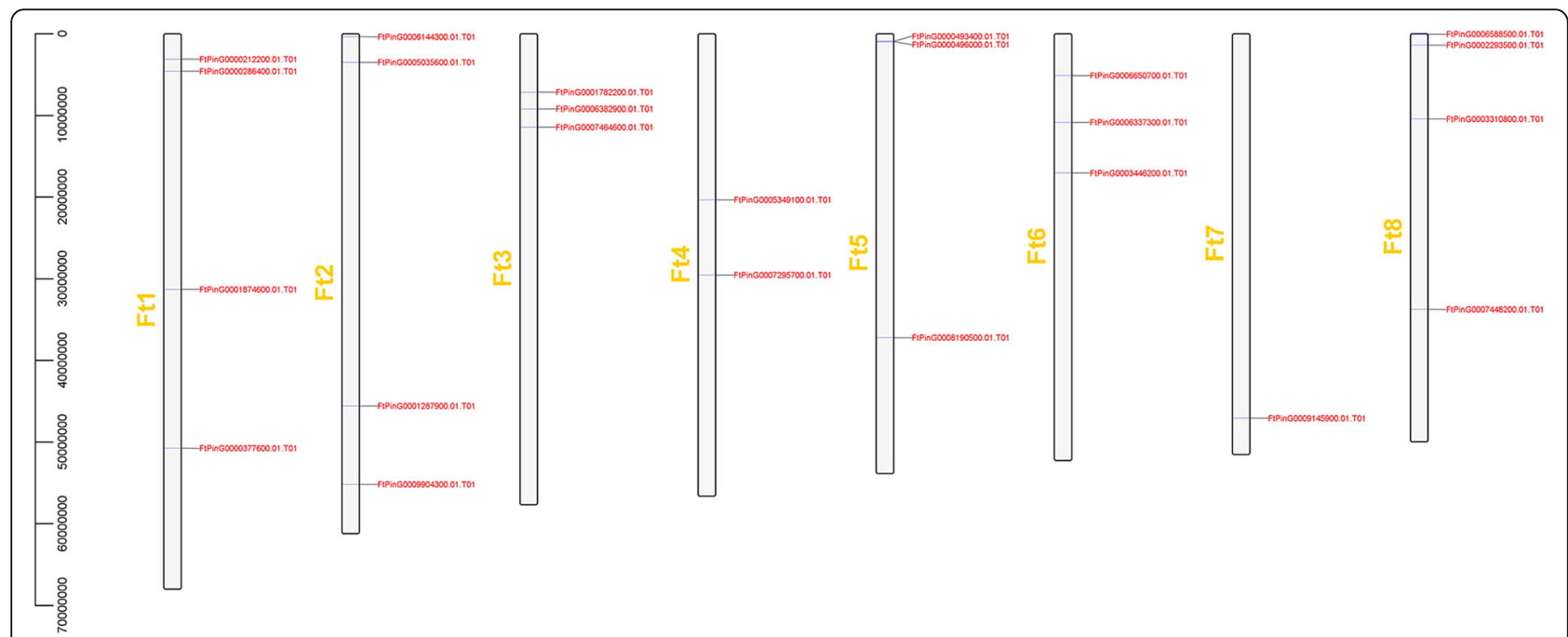

Fig. 3 Schematic representations of the chromosomal distribution of the tartary buckwheat SPL genes. The chromosome number is indicated to the left of each chromosome. 


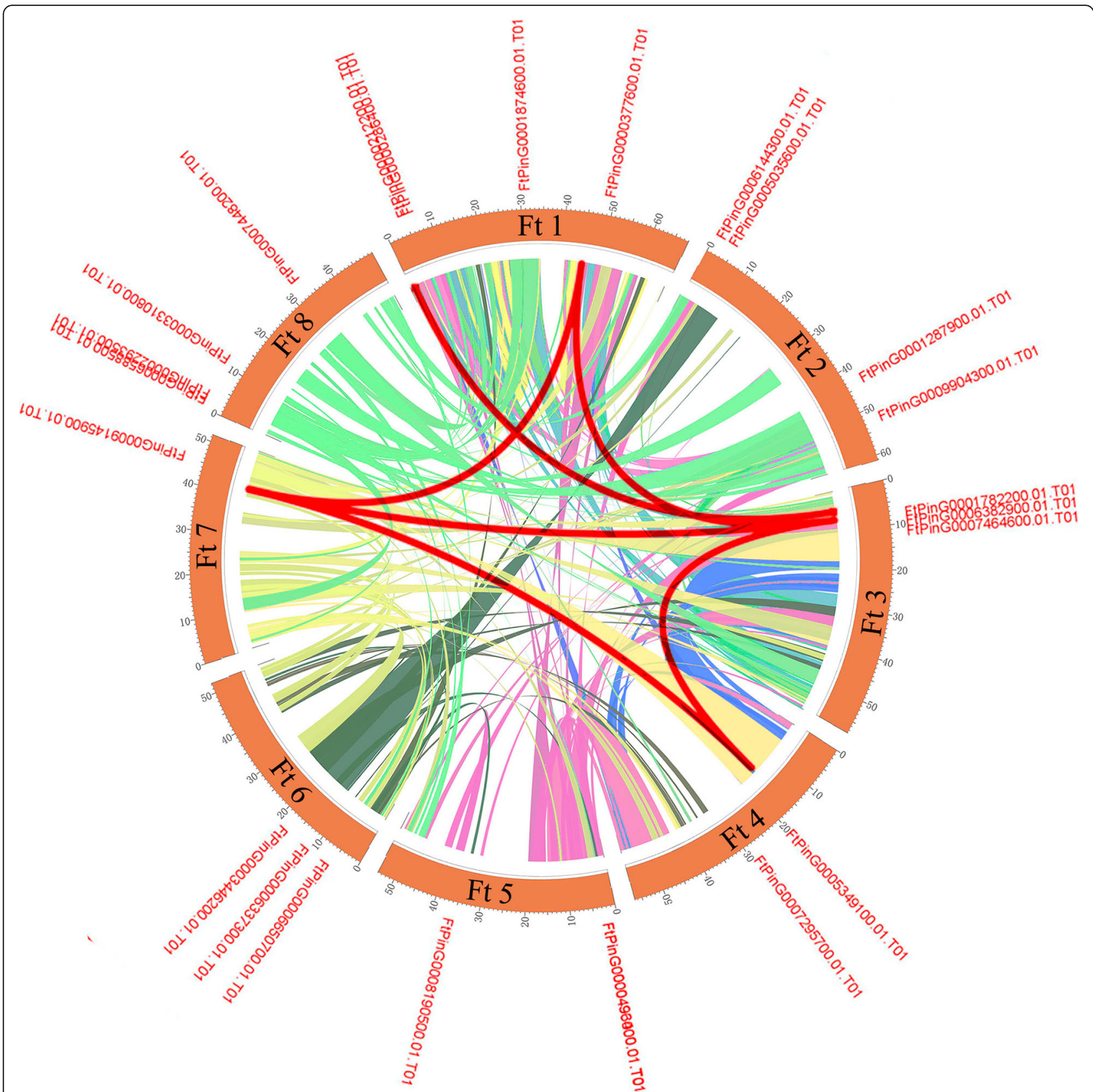

Fig. 4 Schematic representations of the interchromosomal relationships of the tartary buckwheat SPL genes. Colored lines indicate all syntenic blocks in the tartary buckwheat genome.

conclusion, based on the above results, some FtSPL genes may have been produced by segmental duplications, and these replication events are the main driving force of FtSPLS evolution.

\section{Evolutionary analysis of the FtSPL genes and the SPL genes of several different species}

The number of SPL genes identified in tartary buckwheat was similar to that in Salvia miltiorrhiza, apple, maize and Chinese cabbage rice, but the genome size of these five species was very different (tartary buckwheat, $516 \mathrm{Mb}$; Salvia miltiorrhiza, $641 \mathrm{Mb}$; apple, $742 \mathrm{Mb}$; maize, $2300 \mathrm{Mb}$; Chinese cabbage rice, $485 \mathrm{Mb}$ ). [21, 23, 25, 28]. This result indicated that the number of SPL family genes was relatively stable in different species during long-term evolution.

To deduce the evolutionary relationship of the SPL genes, a phylogenetic tree analysis was performed for six dicotyledonous plants (A. thaliana, G. max, B.vulgaris, $S$. lycopersicum, $V$. vinifera, and tartary buckwheat) and a monocotyledonous plant Oryza sativa. From Fig. 5, we 


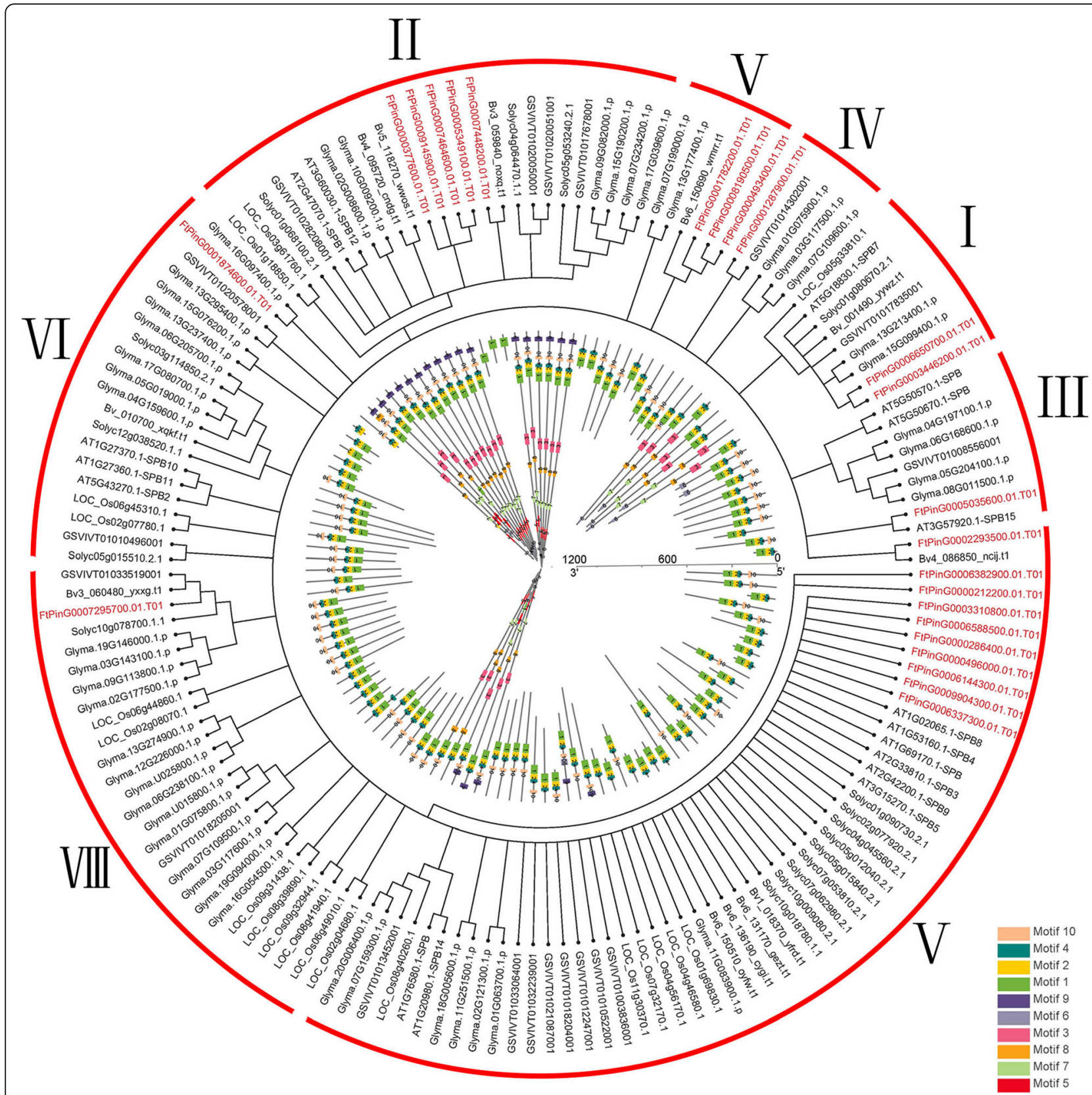

Fig. 5 Phylogenetic relationships and motif compositions of the SPL proteins from seven different plant species. Outer layer: An unrooted phylogenetic tree constructed using Geneious R11 with the neighbor-joining method. Inner layer: Distribution of the conserved motifs in SPL proteins. The differently colored boxes represent different motifs and their positions in each SPL protein sequence.

saw that the SPL proteins were divided into 8 groups by the phylogenetic tree, of which group VI had the largest number of members. A total of 10 conserved motifs were detected in the SPL protein sequences of all plants (Additional file 3 Table S2). With the exception of FtPinG0009145900.01, FtPinG0007464600.01 and FtPin G0005349100.01 only containing motif 1, all other genes contained motif 1 , motif 2 and motif 4 . All conserved motifs were detected in most SPL genes from the other plants in Group II, while several FtSPL genes only contained motif 1 . Overall, SPL genes in the same group of plants had similar motif compositions (Fig. 5).

We performed a syntenic analysis of the $S P L$ genes in six dicotyledonous plants (A. thaliana, B. vulgaris, G. max, $S$. lycopersicum, $V$. vinifera and Tartary buckwheat) and a monocotyledonous plant $O$. sativa to speculate on the evolutionary origin of SPL genes. The SPL genes in tartary buckwheat were homologous to genes in the reference plants, and the highest level of syntenic conservation was observed among G. $\max$, (28 pairs orthologous 
gene pairs distributed throughout all LGs except LG3, LG8, LG9, LG14, LG16, and LG20), $V$. vinifera (24 orthologous gene pairs distributed on LG1, LG5, LG7, LG10, LG12, LG14, LG15, LG17 and LG19), and S. lycopersicum (18 orthologous gene pairs distributed on LG1, LG2, LG3, LG5, LG7 and LG10) (Fig. 6). In the syntenic analysis of the SPL genes of tartary buckwheat and Glycine max, FtPinG0006588500.01 was found to be associated with at least three syntenic gene pairs, suggesting that FtPin G0006588500.01 might play an important role in SPL family evolution (Additional file 4. Table S3). In general, these results indicated that the tartary buckwheat SPL gene family was highly conserved and that the tartary buckwheat SPL genes were closer to the G. $\max$ genes than to $A$. thaliana genes. The SPL genes might have evolved from a common ancestor of the different plants.

\section{Expression patterns of the FtSPL genes in different plant tissues}

To further study the physiological functions of the $S P L$ genes, we measured the expression patterns of each $S P L$ gene in different tissues (roots, stems, leaves, flowers and fruits) of tartary buckwheat by qRT-PCR assay. From the experimental results, we saw that the expression pattern of each gene varied greatly in the different tissues. Among them, $83.3 \%$ of the genes ( 20 genes) were expressed in all tissues, 10 genes (FtPinG0000286400.01/5035600.01/12879 00.01/496000.01/9904300.01/5349100.01/6588500.01/6382 $900.01 / 7464600.01 / 1782200.01$ ) had the highest expression level in the flowers, and the highest expression levels of 3 genes (FtPinG0006337300.01/3310800.01/0493400.01 were found in leaves (Fig. 7). FtPinG0005035600.01 was not expressed in the stems and fruits, FtPinG0002293500.01 was not expressed in the fruits, and FtPinG0009904300.01 was not expressed in the roots. In addition, 10 genes had relatively high expression levels in all tested tissues, including FtPinG0003310800.1, FtPinG0000286400.1, FtPinG000 0496000.1, FtPinG0008190500.1, FtPinG0001287900.1, Ft PinG0006144300.1, FtPinG0000493400.1, FtPinG00063373 00.1, FtPinG0005349100.1, and FtPinG0000212200.1. Amo ng all the FtSPL genes, FtPinG0000286400.1 and FtP inG0006337300.1 showed the highest expression levels in most tissues, with FtPinG0000286400.1 expressed more highly in the flowers, stems, and roots than in other tissues and with FtPinG0006337300.1 expressed more highly in the leaves, stems and flowers than in other tissues. FtPinG0001874600.01 was not expressed in all tissues (not shown in Fig. 7), while the expression levels of FtPinGO 001782200.01 and FtPinG0003446200.01 were low in all tissues. FtPinG0001782200.01 was mainly expressed in the flowers and stems, and FtPinG0003446200.01 was mainly expressed in the roots, flowers and fruits.

In addition, we analyzed the correlations between the expression patterns of the different genes in various tissues.
From the correlation analysis, we saw that the expression patterns of most genes were positively correlated; for example, FtPinG0006382900.01, which was the mostly expressed in the flowers and had a significant positive correlation with many genes (FtPinG0000212200.1/5349100.0 1/0493400.01/1287900.01/8190500.01/0496000.01/1782200 .01) (Fig. 7).

\section{Differential expression of the FtSPL genes during fruit development of tartary buckwheat}

Tartary buckwheat fruit is an important functional food. It has a balanced amino acid composition in its proteins with high biological value [4]. Tartary buckwheat fruit also contains higher crude fiber and vitamin B than other fruits, and the content of rutin in tartary buckwheat is also very high [29]. The growth and development of tartary buckwheat fruit may be regulated by some genes, thus affecting the contents of the nutrients in the fruit. Therefore, we can systematically study the expression of the FtSPL genes at different stages of fruit development (green fruit stage, discoloration stage and initial maturity stage) and find some genes that potentially regulate fruit growth and development.

The expression patterns of the FtSPL genes at different developmental stages (green fruit stage, discoloration stage and initial maturity stage) were different. Twenty-one genes were expressed at all stages of fruit development. With the development of the fruit, the expression levels of most genes (FtPinG0006382900.1, FtPinG0007448200.1, FtPinG0 000496000.1, FtPinG0008190500.1, FtPinG0001287900.1, F tPinG0006588500.1, FtPinG0000377600.1, FtPinG0000286 400.1, FtPinG0000493400.1, FtPinG0009145900.1, FtPinG0 005349100.1, and FtPinG0000212200.1) decreased gradually; the expression levels of three genes (FtPinG00033 10800.1, FtPinG0006144300.1 and FtPinG0006337300.1) were the highest in the discoloration phase, the expression levels of four genes (FtPinG0007295700.1, FtPinG000178 2200.1, FtPinG0009904300.1 and FtPinG0003446200.1) were the lowest in the discoloration stage, and the expression levels of two other genes (FtPinG0007464600.1 and FtPinG0006650700.1) remained stable after the discoloration stage (Fig. 8). Concurrently, we studied the correlations of the FtSPL gene expression patterns with tartary buckwheat fruit development and the correlation of each gene during this process. From the results, we saw that there were three genes (FtPinG0000212200.01/ FtPinG00 08190500.01/ FtPinG0000493400.01) that were significantly negatively correlated with fruit development. Most of the FtSPL genes showed a positive correlation. FtPinG0006 382900.01 was significantly positively correlated with FtPin G0001287900.01 and FtPinG0007448200.01, while FtPinG0 007295700.01 was significantly positively correlated with FtPinG0009904300.01 but significantly negatively correlated with FtPinG0006144300.01 (Fig. 8). 


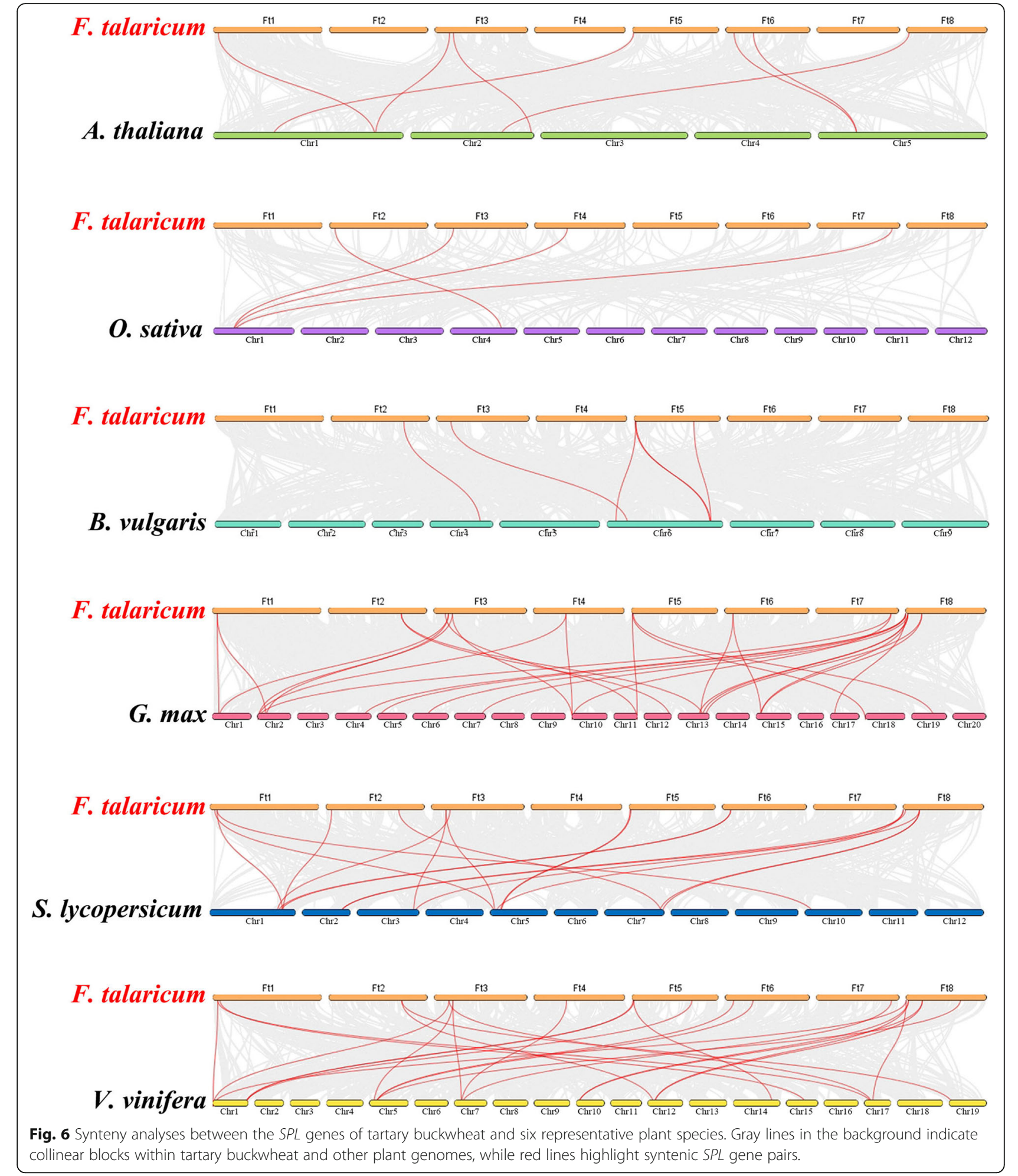

\section{Discussion}

Evolution of the SPL genes in tartary buckwheat

The SPL proteins play an important role in the regulation of plant development, such as in fruit maturation [14, 30], flowering [31] and organ size [32]. The SPL gene family has been isolated and identified from many plants, such as $S$. lycopersicum (15 members), A. thaliana (17 members), $V$. vinifera (18 members) and O. sativa (19 members) [18, 20, 33, 34]. To explore the homology between the SPL genes from $A$. thaliana and rice, a phylogenetic tree showed that 


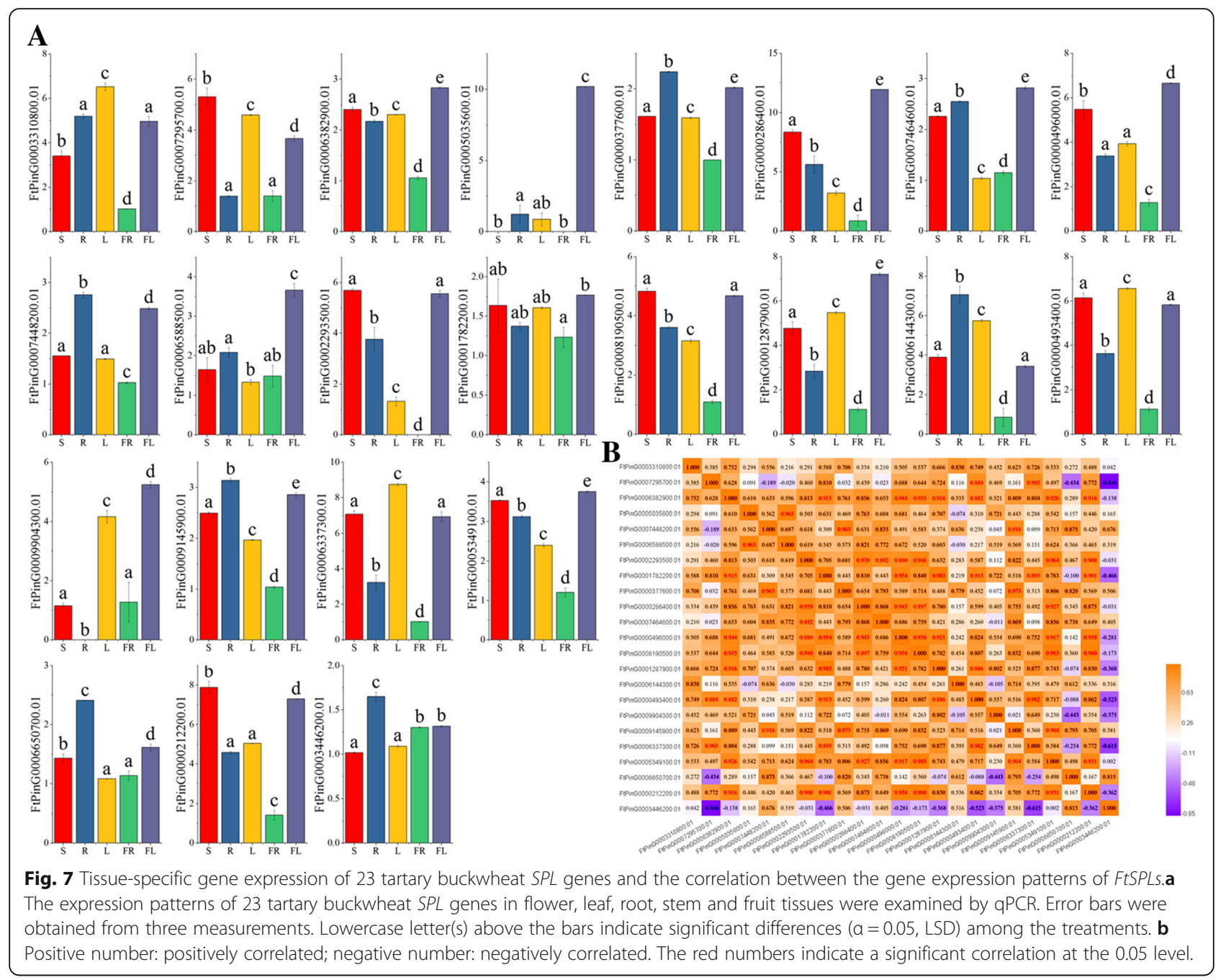

the SPL genes were divided into nine subgroups, while the $S P L$ genes in petunia and $A$. thaliana were divided into eight subgroups $[33,35]$. The 24 FtSPLs identified in this study were also divided into eight subgroups with the genes from $A$. thaliana; each group contained at least one tartary buckwheat SPL gene and A. thaliana SPL gene (Fig. 1). The number of introns in the FtSPL genes ranged from 0 to 10 , and the number of introns and motif compositions within each subgroup were similar, which further supports the grouping of the phylogenetic tree (Fig. 2). The FtSPL genes were distributed unevenly on 8 chromosomes of tartary buckwheat (Fig. 3). When the homology of the SPL genes in the tartary buckwheat genome was analyzed, it was found that there were no tandem duplication gene pairs but 6 pairs of segmental duplications were found (Fig. 4). It is possible that the existence of these homologous genes on the different chromosomes of tartary buckwheat promoted the evolution of the FtSPL genes, which allows the number of SPL genes in tartary buckwheat to be greater than that in other dicotyledonous plants (S. lycopersicum, A. thaliana, $V$. vinifera and $O$. sativa).

To further study the evolutionary relationships of the FtSPL genes, we constructed a phylogenetic tree consisting of six dicotyledonous plants (tartary buckwheat, $A$. thaliana, G. max, B. vulgaris, S. lycopersicum and V. vinifera) and a monocotyledonous plant O. sativa (Fig. 5). The information we obtained from the phylogenetic tree was that the SPL genes from the different plants were also divided into eight groups and that the tartary buckwheat SPL genes were scattered among these eight groups. The FtSPL genes from group I and group VII were clustered with SPL genes in G. $\max$, and the SPL genes from the other groups were clustered with $S P L$ genes in $V$. vinifera, $B$. vulgaris and $A$. thaliana. A syntenic analysis also showed that the FtSPLs had the highest number of homologous gene pairs with G. $\max , V$. vinifera and other dicotyledonous plants. When analyzing the motif compositions of the SPL genes in many plants, we found that although the motif composition of 


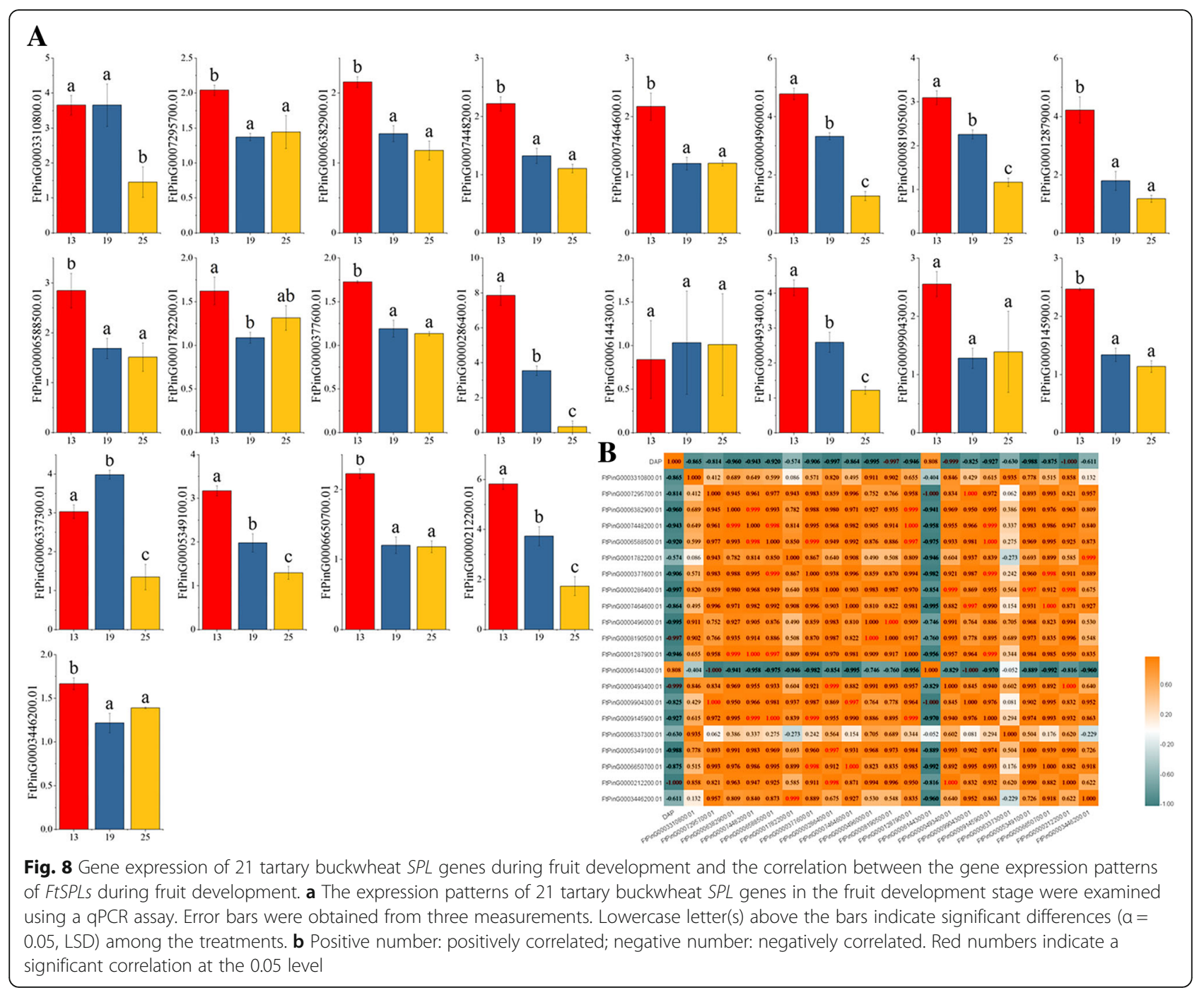

each subgroup was different, the motif composition within the same group was similar, and almost all the members of group II from all plants contained 10 motifs. These results indicated that the SPL genes of tartary buckwheat were closely related to those of the dicotyledonous plants and may have come from the common ancestor.

\section{Expression patterns and functional prediction of the FtSPL genes}

At present, there is little functional data on SPL genes in tartary buckwheat. Usually, a gene performs a function based on the presence of its expression and to a large extent, the expression patterns of genes are related to the functions of the genes [35]. Transcription factors usually play a key role in controlling the expression of tissuespecific genes [36-38]. In this study, the expression pattern of each FtSPL gene was detected by qRT-PCR, and the expression level of each gene was distinct in different tissues (Fig. 7). Most of the FtSPL genes were significantly expressed in the flowers, which is similar to the high expression level of SPL genes from other species in inflorescences and flower buds [18, 34]. Some genes in the same group showed similar expression profiles; for example, many of the genes in group VI had the highest expression in the flowers (FtPinG0006382900.01/FtPinG000658850 0.01/FtPinG0000286400.01/FtPinG0000496000.01/FtPinG 0009904300.01), while genes in group I (FtPinG00066507 00.01/FtPinG0003446200.

01) and in group II (FtPinG0000377600.01/FtPinG 0007448200.01/FtPinG0009145900) were highly expre ssed in the roots (Fig. 7). In addition, FtPinG0006382 900.01 in group VI was significantly positively correlated with the genes (FtPinG0005349100.01/FtPinG00004960 00.01) that were highly expressed in the flowers in the same group. In $A$. thaliana, the transcription level of the SPL15 gene increased during the development process and appeared preferentially in young flowers. Moreover, SPL15 positively regulated the transformation from the 
juvenile phase to the adult phase in the vegetative phase of $A$. thaliana and negatively regulated the formation of leaves in A. thaliana [39]. FtPinG0005035600.01 from group III is a homologous gene of A. thaliana SPL15 (AtSPL15), and it can be seen from Fig. 5 that FtPinG0005035600.01 and AtSPL15 have similar motifs, FtPinG0005035600.01 contains motifs 1, 2, 4, and AtSPL15 contains motifs 1, 2, 4 and 10. Meanwhile, FtPinG0005035600.01 was expressed only in the flowers, roots and leaves, with very high expression in the flowers and very low expression in the leaves (Fig. 5, Fig. 7). Homologous genes may perform similar functions, so in future studies, we can verify whether FtPinGO 005035600.01 has the same function as AtSPL15 through further experiments. AtSPL3 is mainly expressed in inflorescences, and the overexpression of AtSPL3 not only promotes flowering but also leads to abnormal flower and inflorescence development under long periods of sunlight and continuous illumination $[15,40]$. There are many AtSPL3 homologous genes in tartary buckwheat group VI (FtPinG0009904300.01/ FtPinG0000496000.01, etc.) and they are highly expressed in flowers. In future research, we can verify whether these genes (FtPinG0009904300.01/ FtPinG0000496000.01, etc.) have the same function as AtSPL3 through more in-depth experiments.

Tartary buckwheat has a high nutritional value, and its fruits are rich in rutin, which has antioxidant activity [4, 29]. The growth and development of tartary buckwheat will be regulated by many transcription factors [38, 4144]. The regulation of the growth and development of tartary buckwheat by transcription factors will directly affect the nutrient content of tartary buckwheat $[45,46]$. As the main edible part of tartary buckwheat, fruit growth and development processes are particularly important. Therefore, the expression profile of FtSPL genes at different development stages of tartary buckwheat fruits (green fruit stage, discoloration stage and initial maturity stage) can lay a foundation for screening potential genes regulating fruit growth and development. With a qRT-PCR assay, 21 FtSPL genes were found to be expressed in different patterns during the fruit development stages (Fig. 8), indicating that the FtSPL genes may play different roles in fruit growth and development. SPL genes play an important role in the development and maturation of plant fruits, such as AtSPL10, which can affect the length of fruit pods, while in rice, OSSPL16 can control the grain size, shape and quality [14, 47]. In the evolutionary analysis of the FtSPL genes, it was found that FtPinG0007295700.01 and OsSPL16 (LOC_OS08g41940.1) belong to group VII and they have the same motif composition (motifs 1, 2, 4 and 10) (Fig. 5). Previous studies have shown that the green fruit stage is the time at which the fruit size of tartary buckwheat is determined, and the expression level of FtPinG0007295700.01 was the highest in the green fruit stage (Fig. 8). Therefore, in the future research, we can verify whether FtPinG0007295700.01 can also regulate fruit size like OsSPL16 through further more in-depth experiments [48]. LeSPL-CNR (Solyc02g077920.2.1) is a key gene that controls tomato fruit ripening. Epigenetic variation in the promoter region of LeSPL-CNR affects the biosynthesis of carotenoids and cell wall structure, which inhibits the normal ripening of fruit [30, 49]. LeSPL-CNR and tartary buckwheat SPL genes were clustered in group VI, in which FtPinG00063829.00.01, FtPinG000021220 0.01, FtPinG0006588500.01 and FtPinG0000286400.01 had the same motif compositions as LeSPL-CNR, and these FtSPL genes were also highly expressed in the flowers (Fig. 5, Fig. 7). Based on these experimental results, the specific functions of these FtSPL genes can be verified by further experiments in the future.

\section{Conclusion}

These results are the first genome-wide analysis of the $S P L$ gene family in tartary buckwheat.

We comprehensively analyzed 24 putative FtSPL genes, including their gene structures, conserved motifs, gene duplication, evolutionary relationships and spatial and temporal expression patterns, which may be related to their biological functions. The phylogenetic tree divides the FtSPL gene into eight groups, each of which has similar motif compositions and gene structures. The expression patterns of these FtSPL genes in different tissues suggest that these FtSPL genes may regulate the growth and development of tartary buckwheat. Our research lays a foundation for the future elaboration of the potential functions of the FtSPL genes in the growth and development of tartary buckwheat.

\section{Methods}

\section{Plant material}

The tartary buckwheat accessions (XIQIAO) used in this study were requested from Professor Wang Anhu of Xichang University. The materials were planted in the experimental field of the College of Life Science, Sichuan Agricultural University (Lat. 29 $97^{\prime} \mathrm{N}, 102^{\circ} 97^{\prime} \mathrm{E}$, Alt. $580 \mathrm{~m}$ ), Ya'an, Sichuan, China. The samples including flowers, fruits from three $(13,19$, and 25 days after pollination, DAP) different developmental fruit stages, and the stems, roots, and leaves of mature tartary buckwheat were collected separately, quickly put into liquid nitrogen and stored at $-80^{\circ} \mathrm{C}$ for further use.

\section{Identification of the SPL genes in tartary buckwheat}

The tartary buckwheat genome was downloaded from the Tartary Buckwheat Genome Project (TBGP; http://www. mbkbase.org/Pinku1/). The largest number of SPL genes was screened from the tartary buckwheat genome by two BLASTp methods, and the Hidden Markov Model (HMM) profiles corresponding to the SBP domain (PF03110) were 
downloaded from the Pfam protein family database (http://pfam.xfam.org/). The detailed method is as follows: we downloaded the amino acid sequences of all SPL proteins in $A$. thaliana from tair library, which range from 131aa to 1035aa. We identified all similar SPL genes from tartary buckwheat genome by using $S P L$ gene sequences in A. thaliana as a target. After that, we analyzed the conserved domain of FtSPL genes, removed the gene that did not contain SBP conserved domain, and finally 24 genes containing the SBP domain were screened from the tartary buckwheat genome. The PIs, MVs and subcellular localizations of the FtSPL genes were analyzed on the ExPASy website (https://web.expasy.org/compute_pi/).

\section{Phylogenetic analyses and intron-exon structure determination}

The SPL protein sequences (A. thaliana, B. vulgaris, $G$. max, S. lycopersicum, $V$. vinifera and $O$. sativa) for $\mathrm{N}-\mathrm{J}$ phylogenetic trees were downloaded from the UniProt database (https:/www.uniprot.org). Multiple amino acid sequences of identified SPL genes were aligned using Clustalx1.81 program. The phylogenetic trees comparing tartary buckwheat and multiple species (A. thaliana, $B$. vulgaris, G. max, S. lycopersicum, $V$. vinifera and $O$. sativa) were constructed with the NJ method and the specific parameters were Poisson model and 1000 bootstrap replications. The phylogenetic trees comparing tartary buckwheat and $A$. thaliana were also constructed with Mega 7.0 by the N-J method and parameters above. SPL protein sequences from tartary buckwheat and $A$. thaliana were also aligned using Clustalx1.81 program before the phylogenetic tree was constructed. In addition, the online Gene Structure Display Service (http://gsds.cbi.pku.edu.cn) was used to predict the intron structure by comparing the cDNA of the FtSPL genes with the corresponding genomic DNA sequences. The determination of the conserved motifs in FtSPL proteins was conducted by the MEME online program (http:/meme.nbcr.net/meme/intro.html), and the parameters were set to the optimum mode width of 6 to 200 and the maximum number of motifs of 10 .

\section{Chromosomal distribution and gene duplication of the FtSPL genes}

The method of mapping FtSPL genes to the chromosomes of tartary buckwheat was performed according to Liu et al. [50]. Analysis of the FtSPL gene replication events was conducted using multiple collinear scanning toolkits (MCScanX). The syntenic relationship between the FtSPL genes and SPL genes from selected plants was determined by using Dual Synteny Plotter software (https://github.com/CJ-Chen/TBtools).

\section{Expression analysis of the FtAP2/ERF genes by real-time PCR}

The qRT-PCR primers were designed with primer 3 software (http://frodo.wi.mit.edu/) (Additional file 5 Table S4). The design criteria of primers were as follows: PCR product length range of 100 to 200 , GC content is 50 $60 \%$ and primer melting temperatures range of $62^{\circ} \mathrm{C}$ to $67^{\circ} \mathrm{C}$ were selected. Using the tartary buckwheat histone $\mathrm{H} 3$ gene as an internal reference gene, SYBR Premix Ex Taq II (TaKaRa) was used to carry out qRT-PCR [50]. Each experiment was repeated at least three times, and the data were analyzed by the $2^{-(\Delta \Delta C t)}$ method [51].

\section{Statistical analysis}

All the data were analyzed by analysis of variance using the Origin Pro 2018b (OriginLab Corporation., Northampton, Massachusetts, USA) statistics program, and the means were compared by the least significant difference test (LSD) at significance levels of 0.05 and 0.01 .

\section{Additional files} Additional file 1: Figure S1. Alignment of multiple FtSPL and select
SBP domain amino acid sequences. (DOCX $799 \mathrm{~kb}$ )

Additional file 2: Table S1. List of the 134 FtAP2/ERF genes identified in this study. (XLS $114 \mathrm{~kb}$ )

Additional file 3: Table S2. Analysis and distribution of the conserved motifs in tartary buckwheat AP2/ERF proteins. (XLS 36 kb)

Additional file 4: Table S3. One-to-one orthologous relationships between tartary buckwheat and other plants. (XLS 72 kb)

Additional file 5: Table S4. The primer sequences for qRT-PCR. (XLS 33 $\mathrm{kb})$

\section{Abbreviations}

A. thaliana: Arabidopsis thaliana; ABA: Abscisic acid; B. vulgaris: Beta vulgaris; C. annuum: Capsicum annuum L; CDS: Coding sequence; DAP: Days after pollination; Ft: Fagopyrum tataricum; FtSPL: Fagopyrum tataricum SPL; G. max: Glycine max; HMM: Hidden Markov Model; LG: Linkage group; LSD: least significant difference; M. domestica: Malus domestica Borkh; MVs: Molecular weights; NLS: Localization signal; O. sativa: Oryza sativa; Pls: Isoelectric points; qRT-PCR: Quantitative real-time polymerase chain reaction; $R$. communis L: Ricinus communis L; S. Iycopersicum: Solanum lycopersicum; S. miltiorrhiza: Salvia miltiorrhiza; SQ-UA: SQUAMOSA; TBGP: Tartary buckwheat genome project; TFs: Transcription factors; V. vinifera: Vitis vinifera; $Z$. mays: Zea mays $L$

\section{Acknowledgements}

We thank all the colleagues in our laboratory for providing useful discussions and technical assistance. We are very grateful to the editor and reviewers for critically evaluating the manuscript and providing constructive comments for its improvement.

\section{Authors' contributions}

M.-Y.L. planned and designed the research and analyzed data. W.-J.S. wrote the manuscript. W.-J.S., Z.-T.M., L.H., Q.W., Z.-Z.T., T.-L.B., and C.-L.L. performed the experiments. H.C. supervised the research. M.-Y.L., and W.-J.S. contributed equally. All authors read and approved the final manuscript.

Funding

This research was supported by the National Natural Science Foundation of China (31500289), and the National Key R\&D Program of China

(2018YFD1000706). Funds were used for the design of the study and 
collection, analysis, and interpretation of data and in writing the manuscript, as well as in the open access payment.

\section{Availability of data and materials}

The genome sequences of tartary buckwheat used for identifying the AP2/ ERF genes in this study were located in the Tartary Buckwheat Genome Project (TBGP; http://www.mbkbase.org/Pinku1/). The tartary buckwheat accession (XIQIAO) materials used in the experiment were supplied by Professor Wang Anhu of Xichang University. The datasets supporting the conclusions of this article are included in the article and its Additional files.

\section{Ethics approval and consent to participate}

The tartary buckwheat accession (XIQIAO) materials used in the experiment were supplied by Professor Wang Anhu of Xichang University. These plant materials are widely used all over the world and no permits are required for the collection of plant samples. The plant materials are maintained in accordance with the institutional guidelines of the College of Life Sciences, Sichuan Agricultural University, China. This article did not contain any studies with human participants or animals and did not involve any endangered or protected species.

\section{Consent for publication}

Not applicable.

\section{Competing interests}

The authors declare that they have no competing interests.

\section{Author details}

${ }^{1}$ College of Life Science, Sichuan Agricultural University, Ya'an, China. ${ }^{2}$ School of Agriculture and Biolog, Shanghai Jiao Tong University, Shanghai, China.

\section{Received: 30 November 2018 Accepted: 2 July 2019}

Published online: 08 July 2019

\section{References}

1. Takanori O, Kyoko Y, Ohmi O. Two new Fagopyrum (Polygonaceae) species, F. gracilipedoides and F. jinshaense from Yunnan, China. Jpn J Genet. 2002; 77(6):399-408

2. Anton B, Sergey N, Dmitry A, Gurevich AA, Mikhail D, Kulikov AS, Lesin VM, Nikolenko SI, Son P, Prjibelski AD. SPAdes: a new genome assembly algorithm and its applications to single-cell sequencing. J Comput Biol. 2012;19(5):455-77.

3. Eggum BO, Kreft I, Javornik B. Chemical composition and protein quality of buckwheat ( Fagopyrum esculentum Moench ). Plant Foods Hum Nutr. 1980;30(3-4):175-9.

4. Bonafaccia G, Marocchini M, Kreft I. Composition and technological properties of the flour and bran from common and tartary buckwheat. Food Chem. 2003;80(1):9-15.

5. Lee CC, Shen SR, Lai YJ, Wu SC. Rutin and quercetin, bioactive compounds from tartary buckwheat, prevent liver inflammatory injury. Food Funct. 2013; 4(5):794-802.

6. Kamran M, Wennan S, Ahmad I, Xiangping M, Wenwen C, Xudong Z, Siwei M, Khan A, Qingfang H, Tiening L. Application of paclobutrazol affect maize grain yield by regulating root morphological and physiological characteristics under a semi-arid region. Sci Rep. 2018;8(1):4818-33.

7. Liu M, Ma Z, Zheng T, Wang J, Huang L, Sun W, Zhang Y, Jin W, Zhan J, Cai $Y$, et al. The potential role of auxin and abscisic acid balance and FtARF2 in the final size determination of Tartary buckwheat fruit. Int J Mol Sci. 2018; 19(9):2755-74

8. Huijser $\mathrm{P}$, Klein J, Lönnig WE, Meijer H, Saedler H, Sommer H. Bracteomania, an inflorescence anomaly, is caused by the loss of function of the MADSbox gene squamosa in Antirrhinum majus. EMBO J. 1992;11(4):1239-49.

9. Klein J, Saedler HP. A new family of DNA binding proteins includes putative transcriptional regulators of the ANTIRRHINUM MAJUS floral meristem identity gene SQUAMOSA. Mol Gen Genet MGG. 1996;250(1):7-16.

10. Birkenbihl R, Jach $G$, Saedler $H$, Huijser P. Functional dissection of the plantspecific SBP-domain: overlap of the DNA-binding and nuclear localization domains. J Mol Biol. 2005;352(3):585-96.

11. Kazuhiko Y, Takanori K, Makoto I, Masaru T, Tomoko Y, Takashi Y, Masaaki A, Eiko S, Takayoshi M, Emi N. A novel zinc-binding motif revealed by solution structures of DNA-binding domains of Arabidopsis SBP-family transcription factors. J Mol Biol. 2004;337(1):49-63.

12. Jia-Wei W, Benjamin C, Detlef W. miR156-regulated SPL transcription factors define an endogenous flowering pathway in Arabidopsis thaliana. Cell. 2009;138(4):738-49.

13. Wang $Y$, Zong-Li HU, Yang YX, Chen XQ, Chen GP. Bioinformatics analysis of SBP-box gene family in rice. China Journal of Bioinformatics. 2011;9(1):82-71.

14. Masahito S, Tomotsugu K, Nobutaka M, Masaru OT. Arabidopsis SBP-box genes SPL10, SPL11 and SPL2 control morphological change in association with shoot maturation in the reproductive phase. Plant \& Cell Physiology. 2009;50(12):2133-45

15. Gandikota M, Birkenbihl RP, Höhmann S, Cardon GH, Saedler H, Huijser P. The miRNA156/157 recognition element in the 3' UTR of the Arabidopsis SBP box gene SPL3 prevents early flowering by translational inhibition in seedlings. Plant J. 2010:49(4):683-93.

16. Xu M, Hu T, Zhao J, Park MY, Earley KW, Wu G, Yang L, Poethig RS. Developmental functions of miR156-regulated SQUAMOSA PROMOTER BINDING PROTEIN-LIKE (SPL) genes in Arabidopsis thaliana. PLoS Genet. 2016:12(8):e1006263-92.

17. Cardon G, Höhmann S, Klein J, Nettesheim K, Saedler H, Huijser P. Molecular characterisation of the Arabidopsis SBP-box genes. Gene. 1999:237(1):91-104.

18. Salinas M, Xing S, Höhmann S, Berndtgen R, Huijser P. Genomic organization, phylogenetic comparison and differential expression of the SBP-box family of transcription factors in tomato. Planta. 2012;235(6):1171-84.

19. Tripathi RK, Goel R, Kumari S, Dahuja A. Genomic organization, phylogenetic comparison, and expression profiles of the SPL family genes and their regulation in soybean. Development Genes \& Evolution. 2017;227(2):1-19.

20. Hongmin H, Jun L, Min G, Singer SD, Hao W, Linyong M, Zhangjun F, Xiping W. Genomic organization, phylogenetic comparison and differential expression of the SBP-box family genes in grape. PLoS One. 2013;8(3):e59358-73.

21. Li J, Hou H, Li X, Xiang J, Yin X, Gao H, Zheng Y, Bassett CL, Wang X. Genome-wide identification and analysis of the SBP-box family genes in apple (Malus $\times$ domestica Borkh.). Plant Physiol Biochem. 2013;70:100-14.

22. Zhang SD, Ling LZ. Genome-wide identification and evolutionary analysis of the SBP-box gene family in Castor bean. PLoS One. 2014;9(1):e86688-94.

23. Mao HD, Yu LJ, Li ZJ, Yan Y, Han R, Liu H, Ma M. Genome-wide analysis of the SPL family transcription factors and their responses to abiotic stresses in maize. Plant Gene. 2016;6(C):1-12.

24. Zhang HX, Jin JH, He YM, Lu BY, Li DW, Chai WG, Khan A, Gong ZH. Genome-wide identification and analysis of the SBP-box family genes underPhytophthora capsiciStress in pepper (capsicum annuumL.). Front Plant Sci. 2016;7(151):504-18.

25. Linsu Z, Degang Z, Caili L, Fenjuan S, Lu S. Genome-wide analysis and molecular dissection of the SPL gene family in Salvia miltiorrhiza. J Integr Plant Biol. 2014;56(1):38-50.

26. Takeshi U, Gorou H, Satoshi Y, Hirokazu T. The more and smaller cells mutants of Arabidopsis thaliana identify novel roles for SQUAMOSA PROMOTER BINDING PROTEIN-LIKE genes in the control of heteroblasty. Development. 2009;136(6):955-64.

27. Wu G, Poethig RS. Temporal regulation of shoot development in Arabidopsis thaliana by miR156 and its target SPL3. Development. 2006; 133(18):3539-47.

28. Hua-Wei T, Xiao-Ming S, Wei-Ke D, Yan W, Xi-Lin H. Genome-wide analysis of the SBP-box gene family in Chinese cabbage (Brassica rapa subsp. pekinensis). Genome / National Research Council Canada = Ge?nome / Conseil national de recherches Canada. 2015;58(11):463-77.

29. Nina F, Janko R, Iztok Joze K, Zhuanhua W, Zheng Z, Ivan K. Tartary buckwheat (Fagopyrum tataricum Gaertn.) as a source of dietary rutin and quercitrin. Journal of Agricultural \& Food Chemistry. 2003:51(22):6452-5.

30. Kenneth M, Mahmut TR, Mervin P, Yiguo H, Thompson AJ, King GJ, Giovannoni JJ, Seymour GB. A naturally occurring epigenetic mutation in a gene encoding an SBP-box transcription factor inhibits tomato fruit ripening. Nat Genet. 2006;38(8):948-52.

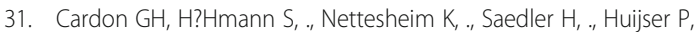
Functional analysis of the Arabidopsis thaliana SBP-box gene SPL3: a novel gene involved in the floral transition. Plant J 2010, 12(2):367-377.

32. Wu G, Park MY, Conway SR, Wang JW, Weigel D, Poethig RS. The sequential action of miR156 and miR172 regulates developmental timing in Arabidopsis. Cell. 2009;138(4):750-9.

33. Yang Z, Wang X, Gu S, Hu Z, Xu H, Xu C. Comparative study of SBP-box gene family in Arabidopsis and rice. Gene. 2008;407(1):1-11. 
34. Kabin X, Congqing W, Lizhong X. Genomic organization, differential expression, and interaction of SQUAMOSA promoter-binding-like transcription factors and microRNA156 in rice. Plant Physiol. 2006;142(1):280-93.

35. Zhou Q, Zhang S, Chen F, Liu B, Wu L, Li F, Zhang J, Bao M, Liu G. Genomewide identification and characterization of the SBP-box gene family in Petunia. BMC Genomics. 2018;19(2):193-211.

36. Ingraham HA, Chen R, Mangalam HJ, Elsholtz HP, Flynn SE, Lin CR, Simmons DM, Swanson L, Rosenfeld MG. A tissue-specific transcription factor containing a homeodomain specifies a pituitary phenotype. Cell. 1988;55(3):519-29.

37. F J N, C M S, M J T. Tissue-specific regulation of the insulin gene by a novel basic helix-loop-helix transcription factor. Genes Dev. 1995;9(8):1009-19.

38. Clevidence DE, Overdier DG, Tao W, ., Qian X, ., Pani L, ., Lai E, ., Costa RH: Identification of nine tissue-specific transcription factors of the hepatocyte nuclear factor 3/forkhead DNA-binding-domain family. Proc Natl Acad Sci U S A 1993, 90(9):3948-3952.

39. Schwarz S, Grande AV, Bujdoso N, Saedler H, Huijser P. The microRNA regulated SBP-box genes SPL9 and SPL15 control shoot maturation in Arabidopsis. Plant Mol Biol. 2008;67(1-2):183-95.

40. Tsubosa Y, Sato H, Tachimori Y, Hokamura N, Hosokawa M, Kinoshita Y, Daiko H, Udagawa H, Ueno M, Seto Y. Functional analysis of the Arabidopsis thaliana SBP-box gene SPL3: a novel gene involved in the floral transition. Plant J. 2010;12(2):367-77.

41. Gao F, Zhou J, Deng RY, Zhao HX, Li CL, Chen H, Suzuki T, Park SU, Wu Q. Overexpression of a tartary buckwheat R2R3-MYB transcription factor gene, FtMYB9, enhances tolerance to drought and salt stresses in transgenic Arabidopsis. J Plant Physiol. 2017:214:81-90.

42. Yao PF, Li CL, Zhao XR, Li MF, Zhao HX, Guo JY, Cai Y, Chen H, Wu Q. Overexpression of a Tartary buckwheat gene, FtbHLH3, enhances drought/ oxidative stress tolerance in transgenic Arabidopsis. Front Plant Sci. 2017;8:625-42.

43. Luo X, Zhao H, Yao P, Li Q, Huang Y, Li C, Chen H, Wu Q. An R2R3-MYB transcription factor FtMYB15 involved in the synthesis of anthocyanin and Proanthocyanidins from Tartary buckwheat. J Plant Growth Regul. 2017; 37(1):1-9.

44. Gao F, Zhao HX, Yao HP, Li CL, Chen H, Wang AH, Park SU, Wu Q. Identification, isolation and expression analysis of eight stress-related R2R3 MYB genes in tartary buckwheat ( Fagopyrum tataricum ). Plant Cell Rep. 2016;35(6):1385-96.

45. Bai YC, Li CL, Zhang JW, Li SJ, Luo XP, Yao HP, Chen H, Zhao HX, Park SU, Wu Q. Characterization of two tartary buckwheat R2R3-MYB transcription factors and their regulation of proanthocyanidin biosynthesis. Physiol Plant. 2014;152(3):431-40.

46. Zhao HX, Wu XF, Bai YC, Li CL, Chen H, Shao JR, Wu Q. Gene expression analysis of key enzymes and MYB transcription factors in flavonoid biosynthesis pathway during germination of Fagopyrum tataricum. Journal of Agricultural Biotechnology. 2012;20(2):121-8.

47. Wang S, Wu K, Yuan Q, Liu X, Liu Z, Lin X, Zeng R, Zhu H, Dong G, Qian Q. Control of grain size, shape and quality by OsSPL16 in rice. Nat Genet. 2012; 44(8):950-4.

48. Liu M, Ma Z, Zheng T, Sun W, Zhang Y, Jin W, Zhan J, Cai Y, Tang Y, Wu Q. Insights into the correlation between physiological changes in and seed development of tartary buckwheat ( Fagopyrum tataricum Gaertn . ). BMC Genomics. 2018;19(1):648-68.

49. Chen W, Kong J, Lai T, Manning K, Wu C, Wang Y, Qin C, Li B, Yu Z, Zhang $X$. Tuning LeSPL-CNR expression by SlymiR157 affects tomato fruit ripening. Sci Rep. 2015;5:7852-8.

50. Liu M, Ma Z, Wang A, Zheng T, Huang L, Sun W, Zhang Y, Jin W, Zhan J, Cai $Y$, et al. Genome-wide investigation of the auxin response factor gene family in Tartary buckwheat (Fagopyrum tataricum). Int J Mol Sci. 2018; 19(11):3526-44.

51. Livak KJ, Schmittgen TD. Analysis of relative gene expression data using real-time quantitative PCR and the $2-\Delta \Delta C T$ method. Methods-A Companion To Methods in Enzymology. 2001;25(4):402-8.

\section{Publisher's Note}

Springer Nature remains neutral with regard to jurisdictional claims in published maps and institutional affiliations.

\section{Ready to submit your research? Choose BMC and benefit from}

- fast, convenient online submission

- thorough peer review by experienced researchers in your field

- rapid publication on acceptance

- support for research data, including large and complex data types

- gold Open Access which fosters wider collaboration and increased citations

- maximum visibility for your research: over $100 \mathrm{M}$ website views per year

At BMC, research is always in progress.

Learn more biomedcentral.com/submissions 